\title{
Central Nervous System Changes in Pelvic Inflammation/Pain Patients
}

\author{
Mohammad Dhafer Asiri ${ }^{1} \cdot$ Rowaa Banjar $^{1} \cdot$ Wadha Al-Qahtani $^{1} \cdot$ Hamidreza Goodarzynejad $^{2} \cdot$ Magdy Hassouna $^{1}$
}

Published online: 2 December 2019

(C) The Author(s) 2019

\begin{abstract}
Purpose of Review Centralized pain syndromes (CPS), including chronic pelvic pain (CPP) syndrome, are significant public health problems with prevalence more than diabetes, cancer, or cardiovascular disease. A variety of pathologies are linked with CPP syndrome; however, pain often continues without the presence of pathology, or when an underlying pelvic disease is found, the extent and severity of pain are disproportionate. Although this is not a systematic review, we performed a detailed literature search to identify relevant papers and to provide the available evidence for central changes in association with CPP syndrome.

Recent Findings Recent advances in brain imaging techniques have provided more accurate data on gray matter volume, functional connectivity, and metabolite levels in the pain-relevant areas of the brain. The present evidence shows that like other chronic pain conditions, the CPP syndrome is associated with central nervous system (CNS) alterations. In particular, these include changes in brain structure, in the activity of both the hypothalamic-pituitary-adrenal (HPA) axis and the autonomic nervous system, and in the behavioral and central response to noxious stimulation.

Summary A growing body of evidence, mostly from neuroimaging, suggests that for many patients with CPP, the pain may be associated to changes in both structure and function of the CNS. The treatment of pain symptoms, even without the presence of identifiable pathology, may prevent the development or at least minimize the progression of long-term central changes. These findings support the use of new therapeutic strategies targeting the CNS for controlling of pain in CPP conditions.
\end{abstract}

Keywords Centralized pain syndromes $\cdot$ Chronic pelvic pain $\cdot$ Neuroimaging $\cdot$ Central sensitization

$\overline{\text { This article is part of the Topical Collection on Inflammatory/Infectious }}$ Bladder Disorders

Magdy Hassouna

Magdy.Hassouna@uhn.com

Mohammad Dhafer Asiri

asirymohd@hotmail.com

Rowaa Banjar

rowaa_banjar@hotmail.com

Wadha Al-Qahtani

wadhaqht90@gmail.com

Hamidreza Goodarzynejad

hamirezg@gmail.com

1 Department of Urology, Toronto Western Hospital (UHN), University of Toronto, 399 Bathurst St., Toronto, ON, Canada

2 Department of Clinical and Experimental Research, Tehran Heart Center, Tehran University of Medical Sciences, Tehran, Iran

\section{Introduction}

"Centralized pain syndromes" (CPS) are associated with more than one specific syndrome in which patients often complain of general pain, mood and sleep disturbances, and fatigue as well as poor quality of life [1]. These syndromes are associated with changes within the central nervous system (CNS) that exaggerate response to pain and/or cause generation of a pain signal to normally non-painful stimuli. They are very common, even more prevalent than diabetes, cancer, or cardiovascular disease $[2 \cdot \bullet]$ and this costs averaged CAD $\$ 16,636$ per patient per year due to health care costs as well as lost productivity, and varies according to the level of long-term pain disability [3]. These conditions markedly affect the patients' daily activity and prompt them to visit physicians. Chronic pelvic pain (CPP) is an example of CPS wherein either the somatic or visceral system or both may be involved in pain perception, making differential diagnosis difficult. Among up 
to one-third of patients, the source of pain in the pelvic region is not recognized, though gynecologic, urologic, gastrointestinal, neurological, and musculoskeletal causes are commonly identified. The urological chronic pelvic pain syndromes (UCPPS) refer to a wide range of urological conditions from CPP syndrome (chronic prostatitis/male CPP syndrome or interstitial cystitis/bladder pain syndrome) to chronic scrotal pain $[4 \cdot, 5]$. Even though there are varying etiologies for these syndromes, they present frequently to urologists and the management can be quite frustrating due to unknown natural history in many cases.

The processing of pain, as a complex cognitive and sensory experience, engages widespread brain regions and neural networks. "Chronic pain," pain lasting or recurring for more than 3 to 6 months, is not simply a prolonged "acute pain." Although variables such as age, genetic susceptibility, and psychosocial factors may play contributing roles, the exact mechanisms that contribute to the development of chronicity from an acute pain process are still unknown $[4 \cdot, 6]$. Despite this, regardless of underlying pathology or pain location, the changes associated with chronicity mechanisms are consistent $[7,8 \bullet, 9 \bullet]$. There are two main theories regarding whether chronic pain conditions are owing to the "bottom-up" or "top-down" mechanisms of pain amplification. According to the bottom-up theory, an excess noxious peripheral input causes an increase in pain perception which in turn eventually sensitizes the CNS to the point of perceiving pain even without the presence of peripheral drive [10]. On the other hand, the top-down theory hypothesizes a brain's descending pain modulatory system in that changes already existed inside the CNS, regardless of peripheral drive, causing perceiving pain [11]. While there are still debates on which theory would best fit in explaining the cause of chronic pain, both of these theories support changes in the CNS pathways.

Our current understanding of the pathophysiology of pelvic inflammation/pain and its related changes in the central nervous system is still incomplete. However, central disturbance in pain processing and in viscerosensory signals is thought to be involved $[12,13]$. The aim of this review is to provide a general overview of existing literature regarding mechanisms within the CNS, which may contribute to the generation and/ or maintenance of pain in conditions associated with CPP syndrome. This might serve as a primer for researchers initiating more thorough investigations for the identification of CNS changes that result in altered pain perception in centralized pain syndromes.

\section{Nociception and Pain}

According to the International Association for the Study of Pain (IASP), nociception and pain are different concepts and should not be confused [14]. Nociception is a neurophysiological term that is defined as a specific activity and objective experience in the neural pathways in response to an adequate stimulus (third-person perspective). Pain, on the other hand, is defined as a subjective experience only felt by the person experiencing it (first-person perspective) that typically accompanies nociception, but may also emerge without any stimulus, and thereby includes the emotional and psychological responses. This distinction has been recently mentioned in a white paper by an IASP task force wherein it is concluded that brain imaging should be used as an adjunct to rather than a replacement for subjective verbal reports of the pain experience [15]. Pain, a product of higher brain center processing, was defined by IASP in 1979 as "an unpleasant sensory and emotional experience associated with actual or potential tissue damage or described in terms of such damage" which is still valid today $[14,16]$.

\section{The Various Types of Urologic Pain}

Due to being subjective experience and its complexity, classifications of pain are complicated and they may overlap. However, pelvic pain, similar to the other types of pain, can be described in terms of underlying mechanism(s) and can also be categorized to "acute" or "chronic" on the basis of pain duration. The biological underlying mechanisms for pelvic pain conditions may include nociceptive, inflammatory, neuropathic, and psychogenic [17••]. When the aforementioned mechanisms overlap, mixed cause of pain happens. In addition, when an etiological factor for pelvic pain is not identified, it is defined as idiopathic pain.

\section{Neuropathic Versus Nociceptive Pain}

Neuropathic pain, or "neuralgia," refers to pain caused by dysfunction of the somatosensory nervous system secondary to trauma, infection, or surgery. Neuropathic pain, which is usually described as a burning, tingling, or shooting pain occurring in the peripheral distribution of the affected nerve, is not a diagnosis but a clinical description; it commonly persists without a demonstrable lesion or an ongoing disease satisfying established neurologic diagnostic criteria. This type of pain can be further divided into three subgroups: (1) sympathetically mediated pain when a peripheral nerve lesion exists, associated with autonomic modifications; (2) peripheral neuropathy when damage to a peripheral nerve exists, without autonomic alterations; and (3) central pain, which may be experienced in conjunction with chronic pain disorders, occurs when CNS changes exist. On clinical examination, there may be associated sensory changes such as hyperalgesia (exaggerated response to a stimulus that normally provokes pain) or allodynia (autonomous generation of pain signals from a stimulus that does not normally provoke pain). 
Whereas with neuropathic pain, sensory changes are confined to the distribution of a specific peripheral nerve, in nociceptive pain, there is generally a specified distribution of pain localizing to an area of tissue damage or inflammation. Nociceptive pain, often considered as "normal pain," refers to pain from noxious stimuli such as tissue damage or inflammation which is caused by direct activation of pain receptors (nocireceptors) in nerve endings inside tissues. It is also provoked by stimuli that would become noxious if prolonged. The injured tissues may release chemicals such as prostaglandins and bradykinins. This type of pain is often described as aching, throbbing, or sharp pain and may be classified based on the source and related nociceptors, or pain detecting neurons. Cutaneous pain (nociceptors terminate just below the skin tissue) is a well-defined, localized pain of short duration owing to the high concentration of nerve endings. Somatic pain (detected with somatic nociceptors originating from tissues, including ligaments, tendons, cartilage, bones, and even blood vessels) is a dull, poorly localized pain of longer duration than cutaneous pain due to scarcity of pain receptors in these areas. Visceral pain (detected with visceral nociceptors located inside the body's organs and internal cavities) is usually more aching and of a longer duration than somatic pain. This type of pain is extremely difficult to localize because of even greater scarcity of nociceptors in these areas. The nociceptive pain in pelvic may be visceral or somatic but it is usually visceral and is provoked by distension, ischemia, or spasm of pelvic organs secondary to thermal, chemical, and mechanical stimuli. Pelvic region is innervated by the visceral (T10-S5) as well as the somatic (T12-S5) nervous system creating a complex anatomical and neurobiological network.

\section{Inflammatory Pain}

Inflammatory pain, that is associated with active inflammatory process as a result of tissue injury, falls within the family of nociceptive pain. The local inflammatory reaction irritates nerve endings [18] and may also activate "sleeping" or "silent" nociceptors which are not normally activated by mechanical or thermal stimuli and do not cause pain except at extreme ranges of intensity. In inflammatory tissue disease (e.g., endometriosis), there is not only an inflammatory pain in response to tissue injury but also many silent visceral nociceptors may respond to mechanical stimuli [19]. There is another mechanism for inflammatory pain, probably involved in pelvic pain, called neurogenic inflammation, in which proinflammatory neuromediators are secreted from nerve fibers [20]. After tissue injury, sleeping nociceptors "wake up" in response to endogenous chemical mediators that can excite or lower thresholds of afferent vagal and afferent nerve nociceptive fibers. Cytokines like interleukin (IL) $-1 \beta$ or tumor necrosis factor (TNF) are main pro-inflammatory mediators with direct impact on central sympathetic activity and nociceptive nerve fibers [20].

\section{Nociceptive and Neuropathic Pain May Coexist}

All nociceptors have the capacity to become more easily excitable (sensitized). A lower activation threshold of nociceptors leads to the development of hyperalgesia with or without allodynia. As a consequence, normally innocuous stimuli may provoke pain. Pelvic pain originated from any of closely related structures (i.e., genitourinary, gastrointestinal, neurological, and musculoskeletal system) might have elements of both neuropathic and nociceptive pain. More than half of women with endometriosis-associated pain, for instance, have a mixed nociceptive-neuropathic picture, though in a small proportion definite neuropathic pain exist [21].

\section{Psychogenic Pain}

The etiology of pelvic pain is not always tissue injury and psychosocial factors have a significant impact on the perception of pain. Pain signals can be amplified or even produced by thoughts, emotions, or psychological conflict. A previous meta-analysis suggested that sexual abuse, anxiety, and depression predispose women to CPP [22]. The physical manifestation of unresolved emotional or psychological conflict may therefore have a role in the etiology of pelvic pain. Factors such as social supports, personal expectations of pain, economic status, work, activity level, and anxiety level may modulate the persistence of pain $[4 \bullet]$.

\section{Acute Versus Chronic Pain}

As noted, the causes of pelvic pain can be categorized by underlying mechanisms and anatomic considerations; however, it is preferable to use the timing of symptom onset for classification.

Similar to the other types of pain, pelvic pain can be categorized to "acute" or "chronic" on the basis of pain duration. Whereas acute pain represents a protective mechanism and an adaptive response to potentially dangerous stimuli [23], CPP represents a pathologic function of the nervous system and is considered a maladaptive response. The CPP is clinically defined as "noncyclic pain of 6 or more months duration that localizes to the anatomic pelvis, abdominal wall at or below the umbilicus, lumbosacral back or the buttocks and is of sufficient severity to cause functional disability or lead to medical care" [24]; it is frequently associated with maladaptive emotional, behavioral, sexual, and cognitive consequences. When chronic pain occurs, neuropathic and inflammatory pains are two principal mechanisms involved in pain perception $[17 \bullet \bullet]$. 


\section{Referred Pain}

Deep visceral pain has some overlap with somatic sensory tracts in the spinal cord, causing "referred pain." The referred pain is a well-known phenomenon since the nineteenth century that is defined by IASP as "pain perceived at a location that is not the origin of the pain" [25]. Referred pain can elicit from a variety of structures, including tendons, ligaments, visceral, skeletal, and myofascial tissues. Although there is an increasing body of literature on the subject, the underlying mechanism is unknown $[26,27]$. Despite this, there are several hypotheses and referred muscle pain may be linked to high sensitivity of specific muscle nociceptors or central sensitization [26].

\section{The CNS Role in Pain Process Modulation}

In UCPPS and other centralized pain syndromes, supraspinal alternations in the CNS such as structural, molecular, and physiological changes lead to pain despite no presence of noxious input from the periphery [28••]. The pain perception could be modulated (amplified or suppressed) through multiple mechanisms. "Endogenous pain inhibition" pathway arising from cortical and spinal mechanisms is an example of pain reduction mechanism [29]. Widespread pain in fibromyalgia and some other chronic pain conditions is related to a deficit of endogenous pain inhibition system [30]. Notably, the "neuromatrix" theory of pain (suggesting that the synaptic architecture of a fixed combination of brain areas or "neuromatrix" determines a characteristic output pattern or "neurosignature" in all individuals) [31], has been debunked by a newer idea of "cerebral signature" [32]—a dynamic brain network which varies between individuals is active in pain perception.

\section{Central Sensitization and Hypothalamic-Pituitary-Adrenal Axis}

The sensory information from major pelvic organs is conveyed via the hypogastric plexus (the main autonomic neuronal center of the pelvis) and pudendal (a major somatic nerve of the sacral plexus) nerves. The cells in thoracolumbar and sacral dorsal root ganglia are the first of several sensory neurons that relay painful sensations from the pelvis to the brain. It is worth noting that sensory information from all pelvic structures may converge on to the same spinal cord neural circuits. The majority of afferent neurons of dorsal root ganglia crosses over (decussates) at the level of the spinal cord and ascends within the spinothalamic tract that synapses to the thalamus. From there, signals go to multiple brain areas that are responsible for the decoding of afferent input and for individual's pain perception. These areas include the primary and secondary somatosensory cortices, cingulated cortex, prefrontal cortex, insular cortex, amygdala, and cerebellum. The sensory input from pain afferent pathways can be augmented or suppressed by these brain circuits and spinal cord descending pathways. The amplification of nociceptive signals in the CNS may contribute to widespread pain. The potential mechanisms of centrally driven pain amplification and their role in increased co-morbidity, poorer pain outcomes, and decreased quality of life among patients diagnosed with CPS are reviewed in detail elsewhere [2••]. Briefly, central sensitization and dysregulation of the hypothalamic-pituitary-adrenal (HPA) axis are two main phenomena that have been suggested to be associated with CPS [2••].

The IASP defines central sensitization as "increased responsiveness of nociceptive neurons in the CNS to their normal or sub-threshold afferent input" [33]; it has been also defined as "an amplification of neural signaling within the CNS that elicits pain hypersensitivity" [9॰]. Sensory hypersensitivity (decreased pain thresholds) and hypoesthesia (increased detection thresholds) are both features of central sensitization, and it presents with symptoms such as hyperalgesia and/or allodynia $[9 \bullet, 34]$. The central sensitization is a central process of the nervous system that encompasses altered sensory processing in the brain, dysfunction of descending pain inhibitory mechanisms, and higher activity of pain facilitatory pathways as well as wind-up (or temporal summation of second pain), and long-term potentiation of neuronal synapses in the anterior cingulate cortex $[2 \bullet \bullet, 35]$. The exact pathophysiology of this phenomenon is unclear, but both top-down and bottom-up mechanisms play an important role in its establishment and maintenance $[2 \bullet \bullet, 35]$. This central hypersensitivity may not only explain the presence of chronic pain in the absence of peripheral pathology in CPP syndromes but also can justify the discrepancy between the magnitude of tissue injury with the magnitude of pain and disability [36].

Abnormal regulation of the HPA axis, as the primary stress response system, is commonly seen in a variety of CPS [2••]. Although acute stress would result in increased HPA axis activity and a higher serum cortisol level, in chronic settings, this response will burnout and the HPA axis is suppressed through mechanisms yet to be fully elucidated [37••]. This may be an adaptive mechanism to counteract further tissue damage both locally and systemically as a consequence of continued activation of fight-flight response systems in the body [38]. However, a low cortisol state in chronic stress can attenuate stress-induced analgesia, which is proposed to facilitate the fight-flight response, and consequently can cause chronic pain disorders $[2 \bullet \bullet, 37 \bullet \cdot]$. Chronic pain, on the other hand, can be considered as a repeated stressor that may cause dysfunction in the HPA axis [39]. It is of note that patients with CPS are more likely to have a history of abuse or neglect and to present with comorbid mood disorders, such as depression, anxiety, or panic disorder [40]. This, at least in part, could be explained 
by the fact that neglect (or mistreatment) in early life not only can permanently alter the function of HPA axis, which its programming occurs early on in development, but also influences the downstream effects of HPA axis on nociceptive processing in the periphery, including increased mast cell infiltration and activation which may result in sensitization of nearby nociceptive afferents and eventually in central sensitization through long-term potentiation in the CNS [41].

\section{Chronic Pain Conditions and Structural Changes in the Brain}

As noted before, it is uncertain whether long-term potentiation in the spinal cord (bottom-up theory) or already existed changes inside the CNS, regardless of peripheral drive (top-down theory), or elements of both cause CPS. However, both of these theories support changes in the CNS pathways that are thought to be key factor for the maintenance of increased pain perception. Brain imaging techniques like structural, diffusion, and functional magnetic resonance imaging (MRI); positron emission tomography (PET); and electro- and magnetoencephalography are now widely used. Among patients with CPS, recent advances in neuroimaging technology have provided more accurate data on gray matter volume, functional connectivity (i.e., communication between brain regions), and metabolite levels in the areas of the brain that are active during the perception of pain (i.e., the primary and secondary somatosensory cortices, cingulated cortex, prefrontal cortex, insular cortex, amygdala, and cerebellum). This is giving us insight into the complexity of the pain experience as well as into the network activity, wiring, and neurochemistry related to the production and modulation of painful experiences [42, 43].

Brain imaging studies in patients with chronic back pain have shown a decrease in gray matter volume in pain-relevant brain areas [44]. In contrast, patients with CPS experience an increase in gray matter volume in similar areas of the brain that is associated with more widespread pain and co-morbidity $[2 \bullet \bullet, 45 \bullet \cdot$. Higher gray matter density within the somatosensory cortex, amygdala, and hippocampus is seen in females with CPP [46-48]. A well-designed study on women with $\mathrm{CPP}$ and endometriosis (E) investigating all four possible combinations (i.e., $\mathrm{CPP}^{-} / \mathrm{E}^{-}, \mathrm{CPP}^{+} / \mathrm{E}^{-}, \mathrm{CPP}^{-} / \mathrm{E}^{+}, \mathrm{CPP}^{+} / \mathrm{E}^{+}$) showed that presence of pelvic pain, regardless of presence or absence of endometriosis $\left(\mathrm{CPP}^{+} / \mathrm{E}^{-}\right.$and $\left.\mathrm{CPP}^{+} / \mathrm{E}^{+}\right)$, reduces gray matter volume in pain-relevant brain regions [49]. In women with endometriosis without pain $\left(\mathrm{CPP}^{-} / \mathrm{E}^{+}\right)$, not only these reductions were absent but also an increased volume of the periaqueductal gray (PAG) was seen [49]. The PAG is one of the key regions of the descending pain inhibitory system (see paragraph below) and acts as an endogenous mechanism of analgesia justifying why these women $\left(\mathrm{CPP}^{-} / \mathrm{E}^{+}\right)$do not experience pain. However, the mechanism(s) underlying alterations in the volume of specific brain regions in chronic pain are not fully understood [7]. Among CPS patients, changes in structure and connectivity are specifically observed in the anterior cingulate cortex and insular cortex $[46,50 \bullet, 51 \bullet]$. The functional connectivity has been found between sensorimotor and insular cortices in patients with UCPPS, again with association to greater widespread pain and lower quality of life scores $[50 \bullet 52 \bullet \bullet$. The UCPPS patients also exhibit abnormal levels of brain metabolites such as choline and gamma aminobutyric acid (GABA) in the anterior cingulate cortex region that is involved in the processing and modulation of pain; again, this is associated with greater functional connectivity and negative mood [52••].

In addition to that anterior cingulate and insular cortices change can cause decreased descending inhibitory control and facilitatory pain signaling, two other major components of the descending pain inhibitory pathway in brainstem, i.e., the "PAG" and "rostral ventromedial medulla (RVM)," may turn off nociceptive signals under normal conditions through their connections into inhibitory interneurons in the spinal cord [53]. Diminished function of the inhibitory interneurons has been suggested as another underlying mechanism for central sensitization [54]. Moreover, RVM may have facilitatory effects on spinal nociceptive transmission and thereby contribute to the perpetuation of hyperalgesic states following peripheral tissue injury [55]. Additionally, long-term potentiation and cortical plasticity are seen following peripheral nerve injury [53] and remodeling of the central nucleus of the amygdala, which is crucial for emotional response to pain and its regulation, occurs as a result of increased peripheral input [56].

\section{CNS-Mediated Cross-Talk and Viscerovisceral Interactions}

The pain originating in one pelvic organ can be transmitted and affect other adjacent organs. Even though the precise mechanism underlying pelvic pain remains still unknown, it is well known that a phenomenon called "neural crosstalk" has a role in the overlap of CPP syndrome [4•,57]. The pelvic organs and structures communicate through nerve connections or reflexes in which pain can also be referred to other organs or areas; for example, some patients diagnosed with interstitial cystitis also have symptoms for inflammatory bowel syndrome and some have also vulvodynia; scrotal pain may switch laterality or become bilateral; or patients with chronic prostatitis/CPP syndrome may develop concomitant scrotal pain. This cross-talk between the organs is complicated and may probably occur in any direction [58]. Therefore, as a result of this cross-talk, on one hand, bladder damage can cause dysmenorrhea and colorectal symptoms. On the other hand, acute infective diarrhea may act as a trigger or initiating factor for bladder symptoms. These cross-organ effects are at least partly centrally mediated $[4 \bullet]$. 
As one of the main factors contributing to the development of CPP syndromes, there may be also visceral cross-sensitization, where there is transmission of noxious stimuli from a diseased pelvic organ to a nearby normal structure, causing functional changes in the latter $[4 \bullet, 17 \bullet \bullet]$. For details, the reader is referred to a recent review on this topic [17••]. This pelvic organ cross-sensitization is thought to be caused by underlying viscerovisceral interactions and reflexes between urinary, genital, and gastrointestinal systems thorough three different interconnected neural pathways: (1) convergence of sensory neural pathways within a dorsal root ganglion, from a diseased pelvic organ to a normal nearby structure; (2) convergence of afferent signals in the spinal cord; and (3) convergence of afferent signals from two different pelvic organs in the brain. Thus, these connections may be peripheral or can involve central mechanisms.

\section{Conclusions}

Chronic pelvic pain can result from a variety of etiologies, including endometriosis, cystitis, and prostatitis. These conditions may have detrimental effects on daily life activities and general well-being, often resulting in symptoms such as depression and anxiety. The UCPPS were historically considered to be due solely to inflammation and tissue injury in the pelvic area. However, a growing body of evidence, mostly from neuroimaging, proposes that for many UCPPS patients, the pain may be linked to the altered structure and function inside the CNS. The phenomenon called as "central sensitization" plays a major role in the development of CPP wherein pain can be both generated and perpetuated by the CNS itself. Similar changes may happen in other chronic pain conditions, including fibromyalgia, irritable bowel syndrome, and chronic fatigue syndrome which are commonly associated with UCPPS. Although in many cases the symptoms may be localized to a single pelvic organ, the CNS involving could lead to a centralized pain syndrome influencing the whole pelvis and as a consequence, causing multiple-organ symptomatology. Therefore, the presence of central changes has the potential to both exacerbate symptoms and predispose the individuals to the development of additional chronic conditions. But it is yet to be determined whether these central changes are a cause, effect, or both of the painful symptoms. Patients with CPP syndrome are often refractory to conventional treatments and therefore, their management is actually challenging for urologists and other physicians [59]. Investigating the supraspinal changes may result in identifying new therapeutic strategies for patients with UCPPS or other chronic pelvic diseases. In addition, while it remains unknown to what extent central changes can be reversed, prompt treatment of pain symptoms, even without the presence of identifiable pathology, may prevent the development or at least minimize the progression of long-term central changes related to chronic pain [59]. It is worth noting that strategies for the relief of symptoms of CPP, which many of them start in adolescence or early adult life, are likely to be of particular importance in this subgroup of patients where the CNS is very plastic and hence potentially more vulnerable to repeated episodes of pain.

\section{Compliance with Ethical Standards}

Conflict of Interest The authors declare that they have no conflict of interest.

Human and Animal Rights and Informed Consent This article does not contain any studies with human or animal subjects performed by any of the authors.

Open Access This article is distributed under the terms of the Creative Commons Attribution 4.0 International License (http:// creativecommons.org/licenses/by/4.0/), which permits unrestricted use, distribution, and reproduction in any medium, provided you give appropriate credit to the original author(s) and the source, provide a link to the Creative Commons license, and indicate if changes were made.

\section{References}

Papers of particular interest, published recently, have been highlighted as:

- Of importance

•• Of major importance

1. Williams DA, Clauw DJ. Understanding fibromyalgia: lessons from the broader pain research community. J Pain. 2009;10(8): 777-91. https://doi.org/10.1016/j.jpain.2009.06.001.

2.• Eller-Smith OC, Nicol AL, Christianson JA. Potential mechanisms underlying centralized pain and emerging therapeutic interventions. Front Cell Neurosci. 2018;12:35. https://doi.org/10.3389/fncel. 2018.00035. Important review on the potential mechanisms of centralized pain.

3. Lalonde L, Choiniere M, Martin E, Berbiche D, Perreault S, Lussier D. Costs of moderate to severe chronic pain in primary care patients - a study of the ACCORD program. J Pain Res. 2014;7:389-403. https://doi.org/10.2147/jpr.s55388.

4. Wu C, Jarvi K. Mechanisms of chronic urologic pain. Can Urol Assoc J. 2018;12(6(Supp13)):S147-S8. https://doi.org/10.5489/ cuaj.5320. Important study on the potential mechanisms of chronic urologic pain.

5. Kleinhans NM, Yang CC, Strachan ED, Buchwald DS, Maravilla KR. Alterations in connectivity on functional magnetic resonance imaging with provocation of lower urinary tract symptoms: a MAPP research network feasibility study of urological chronic pelvic pain syndromes. J Urol. 2016;195(3):639-45. https://doi.org/ 10.1016/j.juro.2015.09.092.

6. Denk F, McMahon SB. Neurobiological basis for pain vulnerability: why me? Pain. 2017;158:S108-S14.

7. May A. Structural brain imaging: a window into chronic pain. Neuroscientist. 2011;17(2):209-20. https://doi.org/10.1177/ 1073858410396220. 
8. Tracey I, Bushnell MC. How neuroimaging studies have challenged us to rethink: is chronic pain a disease? J Pain. 2009;10(11):1113-20. https://doi.org/10.1016/j.jpain.2009.09.001. A critical review presenting data from functional, structural, and molecular imaging studies in patients and animals. This proposes that it might be time to reconsider chronic pain as a disease state.

9. Woolf CJ. Central sensitization: implications for the diagnosis and treatment of pain. Pain. 2011;152(3):S2-S15. An important review addressing the clinical implications of "central sensitization" phenomenon.

10. Price TJ, Gold MS. From mechanism to cure: renewing the goal to eliminate the disease of pain. Pain Med. 2018;19(8):1525-49. https://doi.org/10.1093/pm/pnx108.

11. Harper DE, Schrepf A, Clauw DJ. Pain mechanisms and centralized pain in temporomandibular disorders. J Dent Res. 2016;95(10): 1102-8. https://doi.org/10.1177/0022034516657070.

12. Clemens JQ. Male and female pelvic pain disorders-is it all in their heads? J Urol. 2008;179(3):813-4. https://doi.org/10.1016/j.juro. 2007.12.001.

13. Kilpatrick LA, Kutch JJ, Tillisch K, Naliboff BD, Labus JS, Jiang $\mathrm{Z}$, et al. Alterations in resting state oscillations and connectivity in sensory and motor networks in women with interstitial cystitis/ painful bladder syndrome. J Urol. 2014;192(3):947-55. https:// doi.org/10.1016/j.juro.2014.03.093.

14. Loeser JD, Treede RD. The Kyoto protocol of IASP Basic Pain Terminology. Pain. 2008;137(3):473-7. https://doi.org/10.1016/j. pain.2008.04.025.

15. Davis KD, Flor H, Greely HT, Iannetti GD, Mackey S, Ploner M, et al. Brain imaging tests for chronic pain: medical, legal and ethical issues and recommendations. Nat Rev Neurol. 2017;13(10):624 38. https://doi.org/10.1038/nrneurol.2017.122.

16. Treede RD. The International Association for the Study of Pain definition of pain: as valid in 2018 as in 1979, but in need of regularly updated footnotes. Pain Rep. 2018;3(2):e643. https://doi. org/10.1097/pr9.0000000000000643.

17.• Origoni M, Leone Roberti Maggiore U, Salvatore S, Candiani M. Neurobiological mechanisms of pelvic pain. Biomed Res Int. 2014;2014:903848. https://doi.org/10.1155/2014/903848. Specifically addresses possible neurobiological mechanisms of pelvic pain syndrome by analyzing the neural plasticity and molecules which are involved.

18. McKinnon BD, Bertschi D, Bersinger NA, Mueller MD. Inflammation and nerve fiber interaction in endometriotic pain. Trends Endocrinol Metab. 2015;26(1):1-10. https://doi.org/10. 1016/j.tem.2014.10.003.

19. Howard FM. Endometriosis and mechanisms of pelvic pain. J Minim Invasive Gynecol. 2009;16(5):540-50. https://doi.org/10. 1016/j.jmig.2009.06.017.

20. Pongratz G, Straub RH. The sympathetic nervous response in inflammation. Arthritis Res Ther. 2014;16(6):504.

21. Whitaker LH, Reid J, Choa A, McFee S, Seretny M, Wilson J, et al. An exploratory study into objective and reported characteristics of neuropathic pain in women with chronic pelvic pain. PLoS One. 2016;11(4): 0151950.

22. Latthe P, Mignini L, Gray R, Hills R, Khan K. Factors predisposing women to chronic pelvic pain: systematic review. BMJ. 2006;332(7544):749-55. https://doi.org/10.1136/bmj.38748. 697465.55 .

23. Woolf CJ. Pain: moving from symptom control toward mechanismspecific pharmacologic management. Ann Intern Med. 2004;140(6):441-51.

24. ACOG Practice Bulletin No. 51. Chronic pelvic pain. Obstet Gynecol. 2004;103(3):589-605.

25. Classification of chronic pain. Descriptions of chronic pain syndromes and definitions of pain terms. Prepared by the
International Association for the Study of Pain, Subcommittee on Taxonomy. Pain Suppl. 1986;3:S1-226.

26. Wegener S, Jacobs M. Referred pain. In: Kreutzer JS, DeLuca J, Caplan B, editors. Encyclopedia of clinical neuropsychology. New York: Springer New York; 2011. p. 2134-5.

27. Arendt-Nielsen L, Svensson P. Referred muscle pain: basic and clinical findings. Clin J Pain. 2001;17(1):11-9.

28.• Sadler KE, Kolber BJ, et al. J Urol. 2016;196(1):24-32. https://doi. org/10.1016/j.juro.2015.10.198. Addresses the impact of changes in brain function on bladder pain.

29. Ossipov MH. The perception and endogenous modulation of pain. Scientifica (Cairo). 2012;2012:561761. https://doi.org/10.6064/ 2012/561761.

30. Julien N, Goffaux P, Arsenault P, Marchand S. Widespread pain in fibromyalgia is related to a deficit of endogenous pain inhibition. Pain. 2005;114(1-2):295-302.

31. Melzack R. From the gate to the neuromatrix. Pain. 1999;82:S121S6.

32. Tracey I, Mantyh PW. The cerebral signature for pain perception and its modulation. Neuron. 2007;55(3):377-91. https://doi.org/10. 1016/j.neuron.2007.07.012.

33. Merskey H, Bogduk N. Classification of chronic pain, IASP Task Force on Taxonomy. Seattle: International Association for the Study of Pain Press (Also available online at www.iasp-pain.org); 1994.

34. Malfliet A, Kregel J, Cagnie B, Kuipers M, Dolphens M, Roussel $\mathrm{N}$, et al. Lack of evidence for central sensitization in idiopathic, non-traumatic neck pain: a systematic review. Pain Physician. 2015;18(3):223-36

35. Kaya S, Hermans L, Willems T, Roussel N, Meeus M. Central sensitization in urogynecological chronic pelvic pain: a systematic literature review. Pain Physician. 2013;16(4):291-308.

36. Neziri AY, Haesler S, Petersen-Felix S, Muller M, Arendt-Nielsen L, Manresa JB, et al. Generalized expansion of nociceptive reflex receptive fields in chronic pain patients. Pain. 2010;151(3):798 805. https://doi.org/10.1016/j.pain.2010.09.017.

$37 . •$ Coxon L, Horne AW, Vincent K. Pathophysiology of endometriosisassociated pain: a review of pelvic and central nervous system mechanisms. Best Pract Res Clin Obstet Gynaecol. 2018;51:5367. https://doi.org/10.1016/j.bpobgyn.2018.01.014. An important review on pathophysiology of endometriosis-associated pain with focus on central nervous system mechanisms.

38. Fries E, Hesse J, Hellhammer J, Hellhammer DH. A new view on hypocortisolism. Psychoneuroendocrinology. 2005;30(10):1010-6. https://doi.org/10.1016/j.psyneuen.2005.04.006.

39. Wingenfeld K, Heim C, Schmidt I, Wagner D, Meinlschmidt G, Hellhammer DH. HPA axis reactivity and lymphocyte glucocorticoid sensitivity in fibromyalgia syndrome and chronic pelvic pain. Psychosom Med. 2008;70(1):65-72. https://doi.org/10.1097/PSY. 0b013e31815ff3ce.

40. Nicol AL, Sieberg CB, Clauw DJ, Hassett AL, Moser SE, Brummett CM. The association between a history of lifetime traumatic events and pain severity, physical function, and affective distress in patients with chronic pain. J Pain. 2016;17(12):1334 48. https://doi.org/10.1016/j.jpain.2016.09.003.

41. Burke NN, Finn DP, McGuire BE, Roche M. Psychological stress in early life as a predisposing factor for the development of chronic pain: clinical and preclinical evidence and neurobiological mechanisms. J Neurosci Res. 2017;95(6):1257-70. https://doi.org/10. 1002/jnr.23802.

42. Alger JR, Ellingson BM, Ashe-McNalley C, Woodworth DC, Labus JS, Farmer M, et al. Multisite, multimodal neuroimaging of chronic urological pelvic pain: methodology of the MAPP Research Network. Neuroimage Clin. 2016;12:65-77. https://doi. org/10.1016/j.nicl.2015.12.009. 
43. Lee MC, Tracey I. Imaging pain: a potent means for investigating pain mechanisms in patients. Br J Anaesth. 2013;111(1):64-72. https://doi.org/10.1093/bja/aet174.

44. Apkarian AV, Sosa Y, Sonty S, Levy RM, Harden RN, Parrish TB, et al. Chronic back pain is associated with decreased prefrontal and thalamic gray matter density. J Neurosci. 2004;24(46):10410-5. https://doi.org/10.1523/jneurosci.2541-04.2004.

45.• Kutch JJ, Ichesco E, Hampson JP, Labus JS, Farmer MA, Martucci $\mathrm{KT}$, et al. Brain signature and functional impact of centralized pain: a multidisciplinary approach to the study of chronic pelvic pain (MAPP) network study. Pain. 2017;158(10):1979-91. https://doi. org/10.1097/j.pain.0000000000001001. This is the first report of increased widespread pain being linked with changes in brain structure and function co-occurring in different, but potentially overlapping, clinical chronic pain diagnoses.

46. Martucci KT, Shirer WR, Bagarinao E, Johnson KA, Farmer MA, Labus JS, et al. The posterior medial cortex in urologic chronic pelvic pain syndrome: detachment from default mode network-a resting-state study from the MAPP Research Network. Pain. 2015;156(9):1755-64. https://doi.org/10.1097/j.pain. 0000000000000238

47. Bagarinao E, Johnson KA, Martucci KT, Ichesco E, Farmer MA, Labus J, et al. Preliminary structural MRI based brain classification of chronic pelvic pain: a MAPP network study. Pain. 2014;155(12): 2502-9. https://doi.org/10.1016/j.pain.2014.09.002.

48. Kairys AE, Schmidt-Wilcke T, Puiu T, Ichesco E, Labus JS, Martucci K, et al. Increased brain gray matter in the primary somatosensory cortex is associated with increased pain and mood disturbance in patients with interstitial cystitis/painful bladder syndrome. J Urol. 2015;193(1):131-7. https://doi.org/10.1016/j.juro. 2014.08.042.

49. As-Sanie S, Harris RE, Napadow V, Kim J, Neshewat G, Kairys A, et al. Changes in regional gray matter volume in women with chronic pelvic pain: a voxel-based morphometry study. Pain. 2012;153(5):1006-14. https://doi.org/10.1016/j.pain.2012.01.032.

50. Kutch JJ, Labus JS, Harris RE, Martucci KT, Farmer MA, Fenske $\mathrm{S}$, et al. Resting-state functional connectivity predicts longitudinal pain symptom change in urologic chronic pelvic pain syndrome: a MAPP network study. Pain. 2017;158(6):1069-82. https://doi.org/ 10.1097/j.pain.0000000000000886. The first prospective study looking at the ability of resting-state functional MRI (rsfMRI) to predict short-term longitudinal change in pain symptoms for patients in a state of chronic pain.

51. As-Sanie S, Kim J, Schmidt-Wilcke T, Sundgren PC, Clauw DJ, Napadow V, et al. Functional connectivity is associated with altered brain chemistry in women with endometriosis-associated chronic pelvic pain. J Pain. 2016;17(1):1-13. https://doi.org/10.1016/j. jpain.2015.09.008. A small but important study examining the association between endometriosis-related pelvic pain and altered brain chemistry and function in the pain matrix.

52.• Harper DE, Ichesco E, Schrepf A, Halvorson M, Puiu T, Clauw DJ, et al. Relationships between brain metabolite levels, functional connectivity, and negative mood in urologic chronic pelvic pain syndrome patients compared to controls: a MAPP Research Network study. Neuroimage Clin. 2018;17:570-8. https://doi.org/10.1016/j. nicl.2017.11.014. A cross-sectional but important study suggesting abnormal concentrations of metabolites in brain regions involved in the processing and modulation of pain among female patients with urologic chronic pelvic pain syndrome (UCPPS).

53. Zhuo M. Cortical excitation and chronic pain. Trends Neurosci. 2008;31(4):199-207. https://doi.org/10.1016/j.tins.2008.01.003.

54. Scholz J, Broom DC, Youn DH, Mills CD, Kohno T, Suter MR, et al. Blocking caspase activity prevents transsynaptic neuronal apoptosis and the loss of inhibition in lamina II of the dorsal horn after peripheral nerve injury. J Neurosci. 2005;25(32):7317-23. https://doi.org/10.1523/jneurosci.1526-05.2005.

55. Aslam N, Harrison G, Khan K, Patwardhan S. Visceral hyperalgesia in chronic pelvic pain. BJOG. 2009;116(12):15515. https://doi.org/10.1111/j.1471-0528.2009.02305.x.

56. Cheng SJ, Chen CC, Yang HW, Chang YT, Bai SW, Yen CT, et al. Role of extracellular signal-regulated kinase in synaptic transmission and plasticity of a nociceptive input on capsular central amygdaloid neurons in normal and acid-induced muscle pain mice. $\mathrm{J}$ Neurosci. 2011;31(6):2258-70. https://doi.org/10.1523/jneurosci. 5564-10.2011.

57. Rudick CN, Chen MC, Mongiu AK, Klumpp DJ. Organ cross talk modulates pelvic pain. Am J Phys Regul Integr Comp Phys. 2007;293(3):R1191-8. https://doi.org/10.1152/ajpregu.00411. 2007.

58. Baranowski AP. Chronic pelvic pain. Best Pract Res Clin Gastroenterol. 2009;23(4):593-610. https://doi.org/10.1016/j.bpg. 2009.04.013.

59. Vincent KF CK, Elneil S, Horne AW. Therapies targeting the nervous system for chronic pelvicpain relief. Royal College of Obstetricians and Gynaecologists: Scientific Advisory Committee, RoyalCollege of Obstetricians and Gynaecologists, 2015 Contract No.: 46.

Publisher's Note Springer Nature remains neutral with regard to jurisdictional claims in published maps and institutional affiliations. 